\title{
COLOR MEDICAL IMAGE COMPRESSED USING QUINCUNX WAVELETS AND SPIHT CODING
}

\author{
I.BOUKLI HACENE \\ Tlemcen University ,13000, Algeria . \\ Biomedical Laboratory. \\ Department of electrics and Electronics, Technology Faculty. \\ ismaill80@yahoo.fr \\ M. BELADGHAM \\ Bechar University, 08000, Algeria . \\ Biomedical Laboratory. \\ beladghem@yahoo.fr \\ A. BESSAID \\ Tlemcen University ,13000, Algeria . \\ Biomedical Laboratory. \\ Department of electrics and Electronics, Technology Faculty. \\ a.bessaid@gmail.com
}

\begin{abstract}
In the field of medical diagnostics, interested parties have resorted increasingly to color medical imaging. It is well established that the accuracy and completeness of diagnosis are initially connected with the image quality, but the quality of the image is itself dependent on a number of factors including primarily the processing that an image must undergo to enhance its quality. This paper introduces an algorithm for color medical image compression based on the quincunx wavelets coupled with SPÏHT coding algorithm. In order to enhance the compression by our algorithm, we have compared the results obtained with those of other methods containing wavelet transforms. For this reason, we evaluated two parameters known for their calculation speed. The first parameter is the PSNR; the second is MSSIM (structural similarity) to measure the quality of compressed image. The results are very satisfactory regarding compression ratio, and the computation time and quality of the compressed image compared to those of traditional methods.
\end{abstract}

Keywords: Color Medical image ;Compression ; Quincunx wavelets; PSNR: Peak Signal to Noise Ratio; SSIM: The Structural Similarity Index; SPIHT: Set Partitioning In Hierarchical Trees.

\section{Council for Innovative Research}

Peer Review Research Publishing System

\section{Journal: INTERNATIONAL JOURNAL OF COMPUTERS \& TECHNOLOGY}

\author{
Vol 8, No 2 \\ editor@cirworld.com \\ www.cirworld.com, member.cirworld.com
}




\section{INTRODUCTION}

The massive use of numerical methods in medical imaging (MRI, X scanner, nuclear medicine, etc...) today generates increasingly important volumes of data. The problem becomes even more critical with the generalisation of 3D sequence. So it is necessary to use compressed images in order to limit the amount of data to be stored and transmitted.

Among many compression schemes by transformation have been proposed, we can cite the standards JPEG images, MPEG 1 and 2 for compressing video. All of these standards are based on the discrete cosine transform (DCT). [1]

Over the past ten years, the wavelets (DWT), have had a huge success in the field of image processing, and have been used to solve many problems such as image compression and restoration [2]

However, despite the success of wavelets in various fields of image processing such as encoding, weaknesses have been noted in its use in the detection and representation of the objects' contours. The wavelets transform and other classical multi resolutions decompositions seem to form a restricted and limited class of opportunities for multi-scale representations of multidimensional signals.

To overcome this problem, we propose a new multi resolution decompositions by quincunx wavelets which are better adapted to the image representation. This structure of decomposition allows the construction of a no separable transform. No separable wavelets, by contrast, offer more freedom and can be better tuned to the characteristics of images. Their less attractive side is that they require more computations. The quincunx wavelets are especially interesting because they are nearly isotropic [3]. In contrast with the separable case, there is a single wavelet and the scale reduction is more progressive: one factor instead of 2 .

\section{Quincunx Wavelets}

The separable dyadic analysis require three families of wavelets, which is sometimes regarded as a disadvantage, in addition the factor of addition between two successive scales is 4 which may seem high. It is possible to solve these two problems, but at the cost of the loss of filter separability and therefore a slightly higher computational complexity. An analysis has been particularly well studied to find a practical application, known as "quincunx" , [1], Quincunx decomposition results in fewer subbands than most other wavelet decompositions, a feature that may lead to reconstructed images with slightly lower visual quality.

The method is not used much in practice, but, in [7] presents results that suggest that quincunx decomposition performs extremely well and may be the best performer in many practical situations. Figure (1) illustrates this type of decomposition . [3]

We notice that the dilation factor is not more than 2 between two successive resolutions, and that only one wavelet family is necessary . [8] [9]

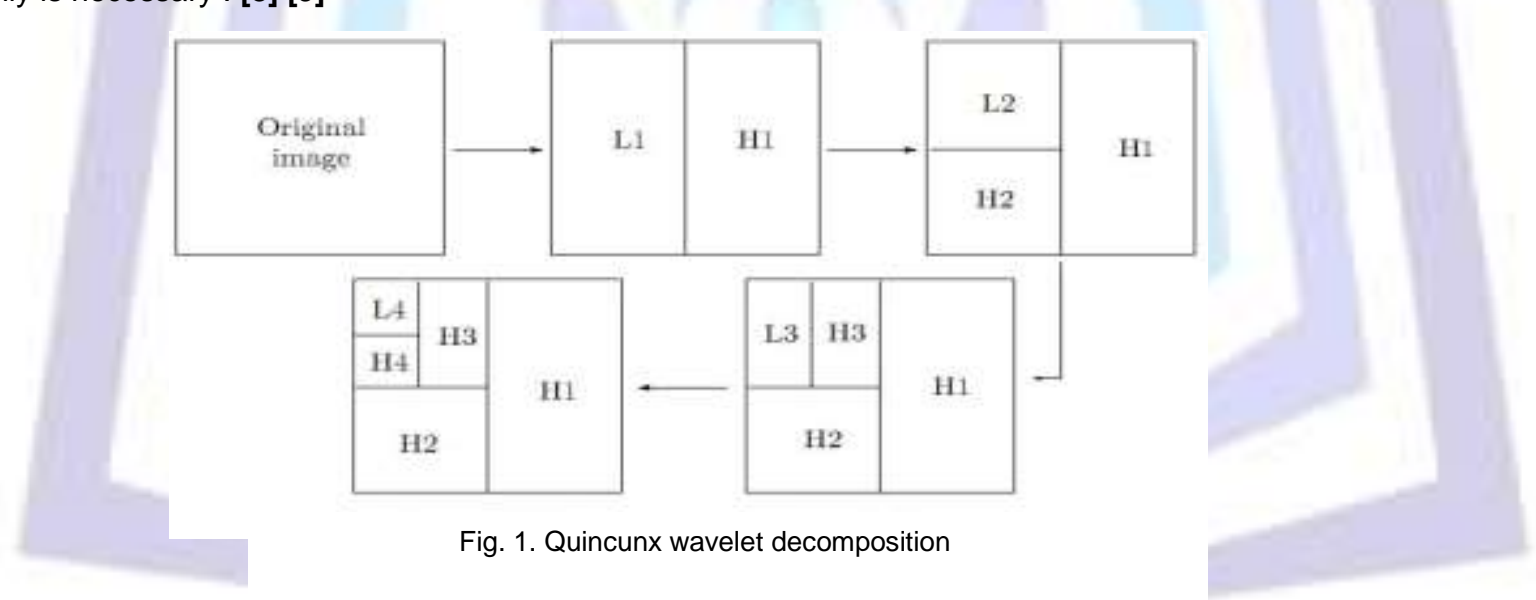

In this case the dilatation matrix will be: $M=\left[\begin{array}{rr}1 & 1 \\ 1 & -1\end{array}\right]$

The Grid transformation (lattice) is done according to the following diagram:

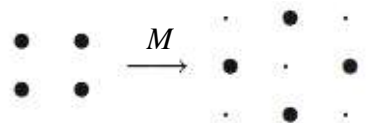

This matrix generates a quincunx lattice in $2 \mathrm{D}$. The column vectors of this matrix form a basis to this lattice. The volume of the unitcell associated equals 2 (Fig. 2). The same lattice is also emanating from the matrix below. [1]

$$
: M^{\prime}=\left[\begin{array}{rr}
1 & -1 \\
1 & 1
\end{array}\right]
$$

It is noticed that the dilatation step is $\sqrt{2}$ on each direction and the geometry of the grid obtained justifies the name given to this multiresolution analysis. 


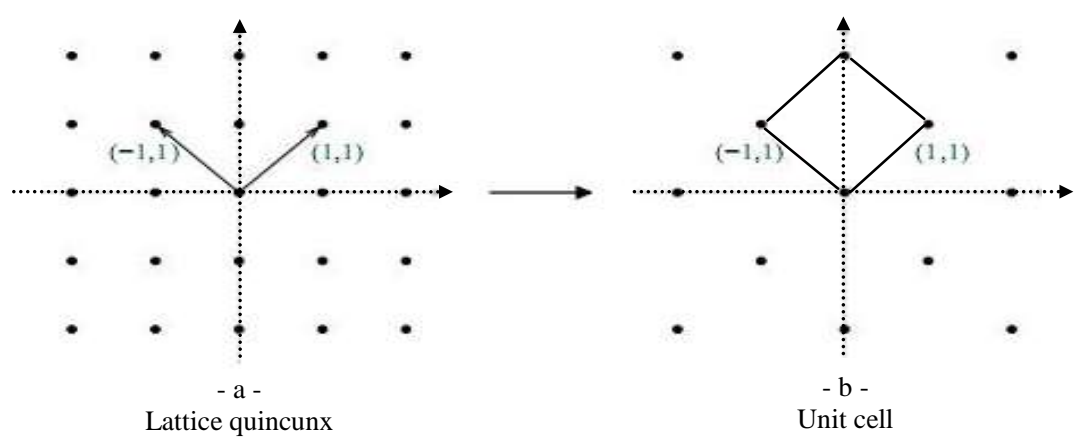

ISSN 22773061

Fig. 2. Examples of a lattice quincunx and unit cell

\section{Quincunx Sampling and Filter banks}

First, we recall some basic results on quincunx sampling and perfect reconstruction filter banks, [10] [11].

The quincunx sampling lattice is shown in figure (3). Let $x[\vec{n}]$ with $\vec{n}=\left(n_{1}, n_{2}\right) \in Z^{2}$ denote the discrete signal on the initial grid. The two-dimensional (2D) z-transform of $x[\vec{n}]$ is denoted by:

$$
X(\vec{z})=\sum_{\vec{n} \in Z^{2}} x[\vec{n}] \vec{z}^{-\vec{n}} \text {, where } \vec{z}^{\vec{n}}=\vec{z}^{n_{1}} \vec{z}^{n_{2}}
$$

The continuous 2D Fourier transform is then given by $X\left(e^{j \vec{\omega}}\right)=\sum_{\vec{n} \in \mathrm{Z}^{2}} x[\vec{n}] e^{-j(\vec{\omega}, \vec{n})}$ with $\vec{\omega}=\left(\omega_{1}, \omega_{2}\right)$ and, finally, the discrete 2-D Fourier transform for $x[\vec{n}]$ given on an $N \times N$ grid $\left(n_{1}, n_{2}=0,1, \ldots, N-1\right)$ by

$$
X[\vec{k}]=\sum_{\vec{n} \in Z^{2}} x[\vec{n}] e^{-j 2 \pi(\vec{k}, \vec{n}) / N}, \text { with }\left(k_{1}, k_{2}=0,1, \ldots, N-1\right)
$$

Now, we write the quincunx sampled version of $x[\vec{n}]$ as:

$$
[x]_{\downarrow M}[\vec{n}]=x[M \vec{n}] \text { where } M=\left(\begin{array}{rr}
1 & 1 \\
1 & -1
\end{array}\right)
$$

Our down-sampling matrix $\mathrm{M}$ is such that $\mathrm{M}^{2}=2 \mathrm{I}$. where I is identity matrix. The Fourier domain version of (1) is

$$
[x]_{\downarrow M}[\vec{n}] \leftrightarrow \frac{1}{2}\left[X\left(e^{j M^{-T} \vec{\omega}}\right)+X\left(e^{j\left(M^{-T} \vec{\omega}+\vec{\pi}\right)}\right)\right]
$$

where $\vec{\pi}=(\pi, \pi)$.

The up-sampling is defined by

$$
[x]_{\uparrow_{M}}[\vec{n}]=\left\{\begin{array}{cc}
x\left[M^{-1} \vec{n}\right], & \text { when } n_{1}+n_{2} \text { is even } \\
0 & \text { elsewhere }
\end{array}\right.
$$

And its effect in the Fourier domain is as follows: $[x]_{\uparrow_{M}}[\vec{n}] \longleftrightarrow X\left(e^{j M^{T} \vec{\omega}}\right)$
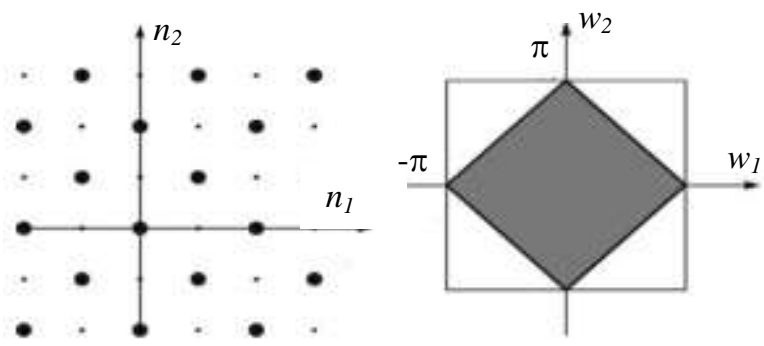

Fig. 3. (a) Quincunx lattice, (b) the corresponding Nyquist area in the frequency domain 


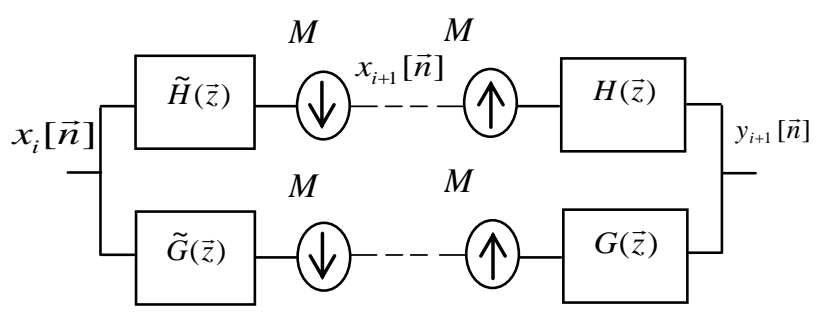

ISSN 22773061

Fig.4. Perfect reconstruction filter bank in a quincunx lattice

If we now chain the down-sampling and up-sampling operators, we get

$$
\begin{gathered}
{[x]_{\downarrow_{M}{ }_{M}}[\vec{n}]=\left\{\begin{array}{cc}
x[\vec{n}], & \text { when } n_{1}+n_{2} \text { is even } \\
0 & \text { elsewhere }
\end{array}\right.} \\
{[x]_{\downarrow_{M \uparrow M}}[\vec{n}]=\frac{1}{2}\left[X\left(e^{j \vec{\omega}}\right)+X\left(e^{j(\vec{\omega}+\vec{\pi})}\right)\right]}
\end{gathered}
$$

Since quincunx sampling reduces the number of image samples by a factor of two, the corresponding reconstruction filter bank has two channels (Fig.4). The low-pass filter $\widetilde{H}$ reduces the resolution by a factor of $\sqrt{2}$; the wavelet coefficients correspond to the output of the high-pass filter $2 \widetilde{G}$.e.g. "see Ref. 8-9-10".

Applying the relation "Eq.(6)" to the block diagram in figure (5), it is easy to derive the conditions for a perfect reconstruction

$$
\left\{\begin{array}{l}
\tilde{H}(\vec{z}) H(\vec{z})+\widetilde{G}(\vec{z}) G(\vec{z})=2 \\
\tilde{H}(-\vec{z}) H(\vec{z})+\tilde{G}(-\vec{z}) G(\vec{z})=0
\end{array}\right.
$$

Where $H$ and $G$ (respectively $\tilde{H}$ and $\widetilde{G}$ ) are the transfer functions of the synthesis (respectively analysis) filters. In the orthogonal case, the analysis and synthesis filters are identical up to a central symmetry; the wavelet filter $G$ is simply a modulated version of the low-pass filter $H$.

\section{Fractional Quincunx Filters}

To generate quincunx filters, we will use the standard approach which is to apply the diamond McClellan transform to map a 1D design onto the quincunx structure . [14]

\section{i. New 1D Wavelet Family}

As starting point for our construction, we introduce a new 1-D family of orthogonal filters

$$
\begin{aligned}
& H_{\alpha}(z)=\frac{\sqrt{2}\left(z+2+z^{-1}\right)^{\frac{\alpha}{2}}}{\sqrt{\left(z+2+z^{-1}\right)^{\alpha}+\left(-z+2-z^{-1}\right)^{\alpha}}} \\
& H_{\alpha}(z)=\frac{\sqrt{2}(2+2 \cos \omega)^{\frac{\alpha}{2}}}{\sqrt{(2+2 \cos \omega)^{\alpha}+(2-2 \cos \omega)^{\alpha}}}
\end{aligned}
$$

Which is indexed by the continuously-varying order parameter $\alpha$.

These filters are symmetric and are designed to have zeros of order $\alpha$ at $z=-1$; the numerator is a fractional power of $\left(z+2+z^{-1}\right)$ (the simplest symmetric refinement filter of order 2 ) and the denominator is the appropriate L2-orthonormalization factor. Also note that these filters are maximally flat at the origin; they essentially behave $H_{\alpha}(z) / \sqrt{2}=1+O\left(\omega^{\alpha}\right)$ as $\omega \rightarrow 0$. Their frequency response is similar to the Daubechies' filters with two important differences: 1) the filters are symmetric and 2) the order is not restricted to integer values. [8] [9]

\section{ii. Corresponding 2D Wavelet Family}

Applying the diamond McClellan transform to the filter above is straightforward; it amounts to replacing cos $w_{\text {by }}$ $(1 / 2)\left(\cos \omega_{1}+\cos \omega_{2}\right)$ in "Eq. (8)". Thus, our quincunx refinement filter is given by 


$$
H_{\alpha}\left(e^{j \vec{\omega}}\right)=\frac{\sqrt{2}\left(2+\cos \omega_{1}+\cos \omega_{2}\right)^{\frac{\alpha}{2}}}{\sqrt{\left(2+\cos \omega_{1}+\cos \omega_{2}\right)^{\alpha}+\left(2-\cos \omega_{1}-\cos \omega_{2}\right)^{\alpha}}}
$$

This filter is guaranteed to be orthogonal because the McClellan transform has the property of preserving biorthogonality. Also, by construction, the $\alpha$ th order zero at $\omega=\pi$ gets mapped into a corresponding zero at $\left(\omega_{1}, \omega_{2}\right)=(\pi, \pi)$; this is precisely the condition that is required to get a 2-D wavelet transform of order $\alpha$. Also, note the isotropic behavior and the flatness of $H_{\alpha}\left(e^{j \vec{\omega}}\right)$ around the origin; i.e, $H_{\alpha}\left(e^{j \vec{\omega}}\right) / \sqrt{2}=1+O\left(\|\vec{\omega}\|^{\alpha}\right)$ for $\vec{\omega} \rightarrow 0$. The orthogonal wavelet filter is obtained by modulation

$$
G_{\alpha}(\vec{z})=z_{1} H_{\alpha}\left(-\vec{z}^{-1}\right)
$$

The corresponding orthogonal scaling function is defined implicitly as the solution of the quincunx two-scale relation

$$
\varphi_{\alpha}(\vec{x})=\sqrt{2} \sum_{\vec{n} \in Z^{2}} h_{\alpha}[\vec{n}] \varphi_{\alpha}(M \vec{x}-\vec{n})
$$

Since the refinement filter is orthogonal with respect to the quincunx lattice, it follows that $\varphi_{\alpha}(\vec{x}) \in L_{2}\left(R^{2}\right)$ and that it is orthogonal to its integer translates. Moreover, for $\alpha>0$, it will satisfy the partition of unity condition, which comes as a direct consequence of the vanishing of the filter at $\left(\omega_{1}, \omega_{2}\right)=(\pi, \pi)$ Thus, we have the guarantee that our scheme will yield orthogonal wavelet bases of $L_{2}\left(R^{2}\right)$. The underlying orthogonal quincunx wavelet is simply

$$
\psi_{\alpha}(\vec{x})=\sqrt{2} \sum_{\vec{n} \in Z^{2}} g_{\alpha}[\vec{n}] \varphi_{\alpha}(M \vec{x}-\vec{n})
$$

\section{Compression Quality Evaluation}

The Peak Signal to Noise Ratio (PSNR) is the most commonly used as a measure of quality of reconstruction in image compression. The PSNR were identified using the following formulae:

$$
M S E^{2}=\frac{1}{M x N} \sum_{1=1}^{i=N} \sum_{j=1}^{j=M}(I(i, j)-\hat{I}(i, j))^{2}
$$

Mean Square Error (MSE) which requires two MxN grayscale images $/$ and $\hat{I}$ where one of the images is considered as a compression of the other is defined as:

- $\quad$ The PSNR is defined as:

$$
P S N R=10 \log _{10}\left(\frac{(\text { Dynamics of image })^{2}}{M S E}\right)
$$

Usually an image is encoded on 8 bits. It is represented by 256 gray levels, which vary between 0 and 255 , the extent or dynamics of the image is 255 .

- The structural similarity index (SSIM):

The PSNR measurement gives a numerical value on the damage, but it does not describe its type. Moreover, as is often noted in [16]-[17] , it does not quite represent the quality perceived by human observers. For medical imaging applications where images are degraded must eventually be examined by experts, traditional evaluation remains insufficient. For this reason, objective approaches are needed to assess the medical imaging quality. We then evaluate a new paradigm to estimate the quality of medical images, specifically the ones compressed by wavelet transform, based on the assumption that the human visual system (HVS) is highly adapted to extract structural information. The similarity compares the brightness, contrast and structure between each pair of vectors, where the structural similarity index (SSIM) between two signals $x$ and $y$ is given by the following expression [18]-[19]:

$$
\operatorname{SSIM}(x, y)=l(x, y) . c(x, y) . s(x, y)
$$

Finally the quality measurement can provide a spatial map of the local image quality, which provides more information on the image quality degradation, which is useful in medical imaging applications. For application, we require a single overall measurement of the whole image quality that is given by the following formula: 


$$
\operatorname{MSSIM}(I, \hat{I})=\frac{1}{M} \sum_{i=1}^{M} \operatorname{SSIM}\left(I_{i}, \hat{I}_{i}\right)
$$

Where $I$ and $\hat{I}$ are respectively the reference and degraded images, $I_{i}$ and $\hat{I}_{i}$ are the contents of images at the i-th local window.

M: the total number of local windows in image. The MSSIM values exhibit greater consistency with the visual quality.

\section{ALGORITHM}

Before applying Quincunx wavelet technique on the retinographic color images, the RGB color images are converts into $\mathrm{YCbCr}$ form, and then applying Quincunx wavelet technique on each layer independently, this means each layer from $\mathrm{YCbCr}$ are compressed as a grayscale image. Figure-5 shows Quincunx wavelet applied on each $\mathrm{YCbCr}$ layer. $\mathrm{YCbCr}$ refers to the color resolution of digital component video signals, which is based on sampling rates. In order to compress bandwidth, $\mathrm{Cb}$ and $\mathrm{Cr}$ are sampled at a lower rate than $\mathrm{Y}$, which is technically known as "chroma subsampling."

This means that some color information in the image is being discarded, but not brightness (luma) information. We obtains the best rate of compression using the rich less layer for the chromatic composante $\mathrm{Cb}$ and $\mathrm{Cr}$.

$$
\left\{\begin{array}{l}
Y=0.2989 \times R+0.5866 \times G+0.1145 \times B \\
C b=-0.1687 \times R-0.3312 \times G+0.5 \times B \\
C r=0.5 \times R-0.4183 \times G-0.0816 \times B
\end{array}\right.
$$

When the decomposition image is obtained, we try to find a way to code the Quincunx wavelet coefficients into an efficient result, taking redundancy and storage space into consideration. After,we apply SPÏHT algorithm on each layer $(\mathrm{y}, \mathrm{Cr}, \mathrm{Cb})$ independently .

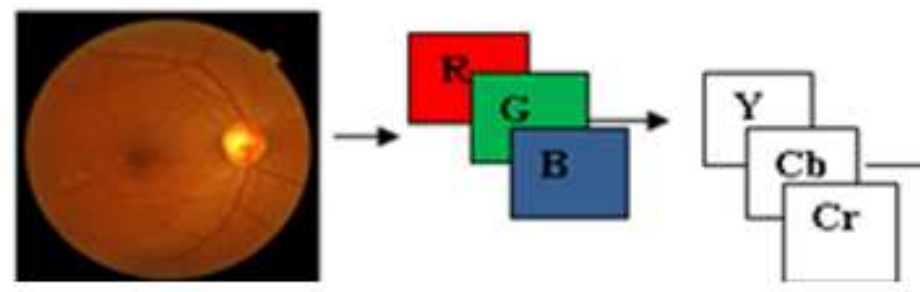

RGB color Image RGB Converted to $\mathrm{YCbCr}$

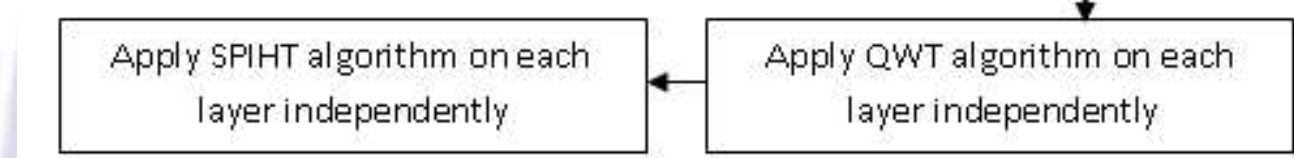

Fig.5. complete steps image compression Technique using Quincunx wavelet(QWT) coupled with SPIHT

This process is repeated for every resolution in the case of level 3 decompositions. The encoding / decoding can be terminated at any time, with the best reproduction obtained up to that point. This is made possible because of the progressive nature of the coding algorithm.

\section{Results and Discussion}

We are interested in lossy compression methods based on 2D wavelet transforms because of their interesting properties. Indeed, the 2D wavelet transforms combines good spatial and frequency locations. As we work on medical image, the spatial location and frequency are important [19]-[20]

In this article we have applied our algorithm to compress medical images. For this reason we have chosen a retinographic image size $512 \times 512$ (color), encoded on 8 bits per pixel. This image is taken from the GE Medical System database [21].

These images were tested on Intel Core (I3) $2.13 \mathrm{GHz}$ PC with 2GB of RAM using Matlab 2009.b 


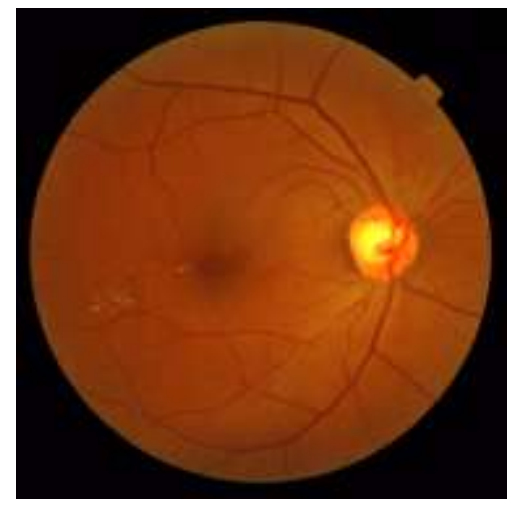

Fig.6- original color image

The importance of our work lies in the possibility of reducing the rates for which the image quality remains acceptable. Estimates and judgments of the compressed image quality are given by the PSNR evaluation parameters and the MSSIM similarity Index.

Figure (7) shown below illustrates the compressed image quality for different bit-rate values (number of bits per pixel). According to the PSNR and MSSIM values, we note that from $0.5 \mathrm{bpp}$, image reconstruction becomes almost perfect.

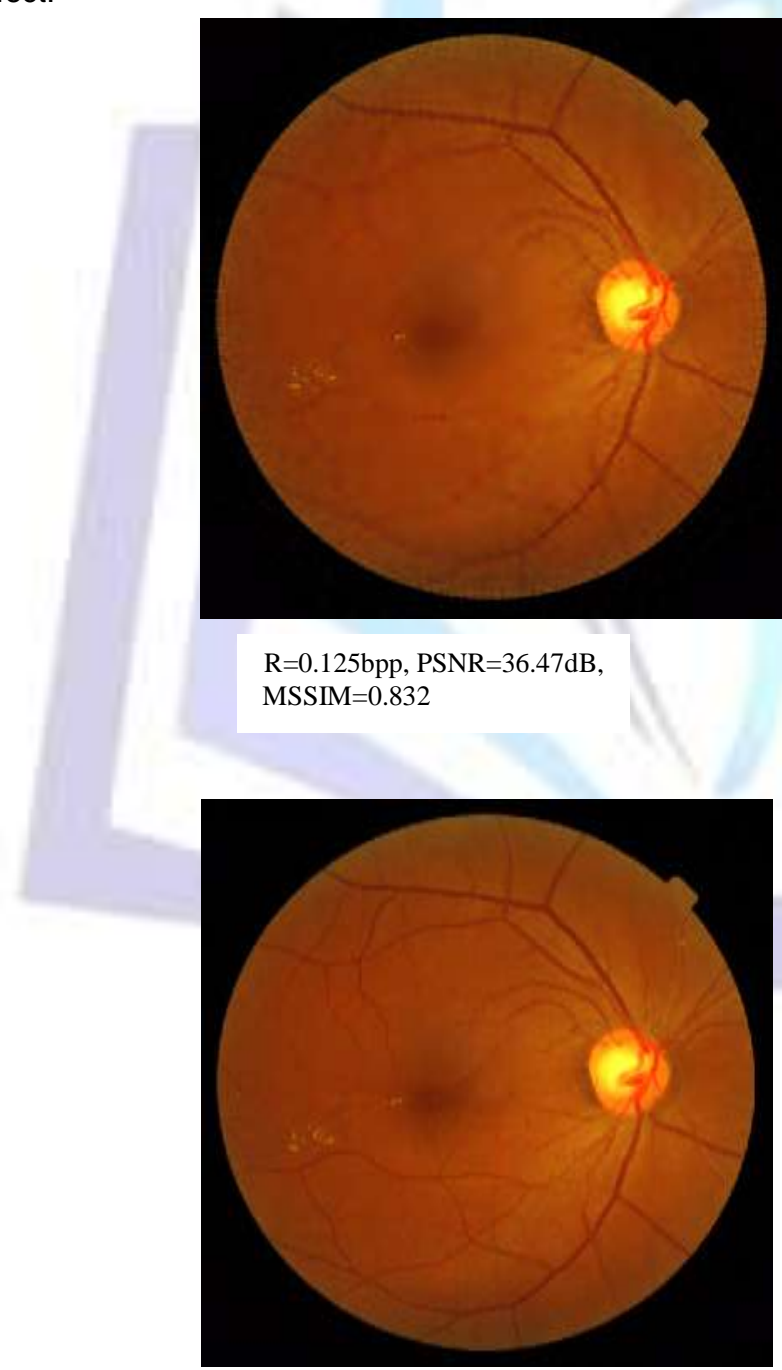

$\mathrm{R}=0.5 \mathrm{bpp}, \mathrm{PSNR}=40.56 \mathrm{~dB}$, MSSIM $=0.918$

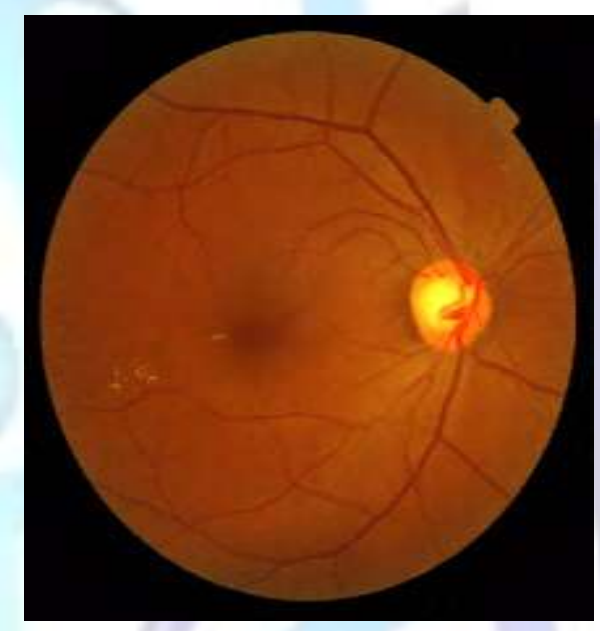

$\mathrm{R}=0.25 \mathrm{bpp}, \mathrm{PSNR}=38.64 \mathrm{~dB}$, MSSIM $=0.88$

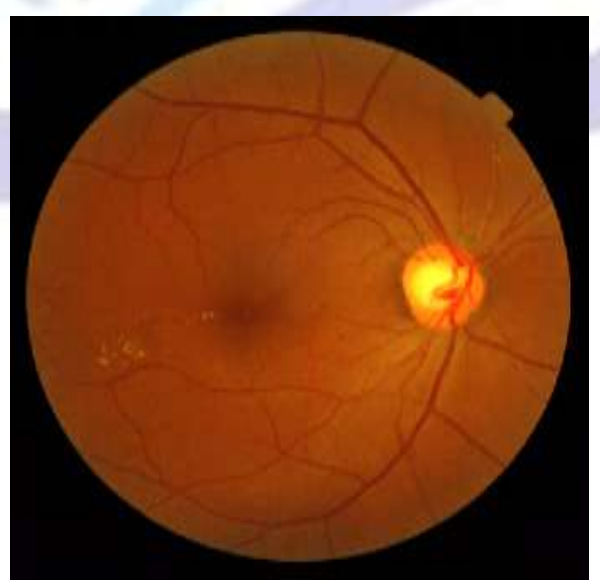

$\mathrm{R}=0.75 \mathrm{bpp}, \mathrm{PSNR}=41.71 \mathrm{~dB}$, MSSIM $=0.938$ 


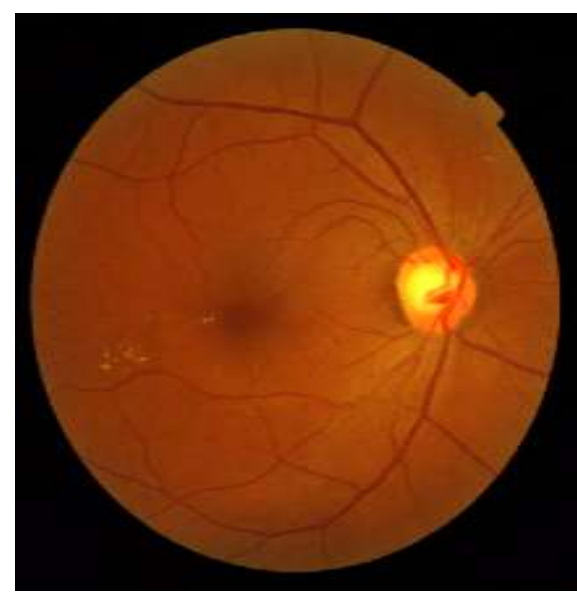

$\mathrm{R}=1 \mathrm{bpp}, \mathrm{PSNR}=42.78 \mathrm{~dB}$, MSSIM $=0.949$

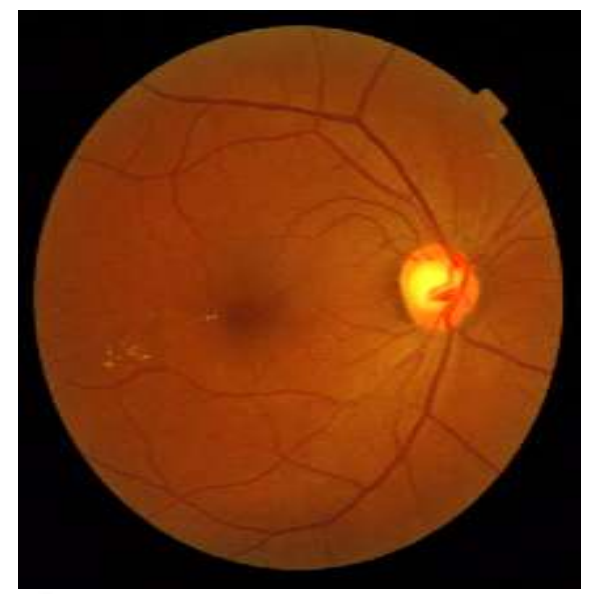

$\mathrm{R}=2 \mathrm{bpp}, \mathrm{PSNR}=46.51 \mathrm{~dB}$, MSSIM=0.971

Fig. 7. Compressing of a Retinographic slice with Quincunx wavelet and SPIHT coding.

To show the performance of the proposed method, we will now make a comparison between these different types of transform (Quincunx wavelet; CDF 9/7 (Filter Bank) and CDF9/7 (Lifting scheme)) coupled with the SPIHT coding and CDF9/7 (Lifting scheme) combined with the EZW coding. For each application we vary the bit-rate 0.125 to 2 and calculate the PSNR and MSSIM. The results obtained are given in Table 1.

Table1. PSNR and MSSIM variation using different methods (Retinographic slice)

\begin{tabular}{lllll}
\hline $\begin{array}{l}\text { Rc } \\
\text { (bpp) }\end{array}$ & \multicolumn{2}{l}{$\begin{array}{l}\text { Quincunx wavelet } \\
\text { +SPIHT }\end{array}$} & $\begin{array}{l}\text { CDF9/7(lifting)+ } \\
\text { SPIHT }\end{array}$ \\
\cline { 2 - 5 } & PSNR & MSSIM & PSNR & MSSIM \\
$\mathbf{0 . 1 2 5}$ & 36.47 & 0.83 & 34.75 & 0.79 \\
$\mathbf{0 . 2 5}$ & 38.64 & 0.88 & 34.97 & 0.81 \\
$\mathbf{0 . 5}$ & 40.56 & 0.91 & 37.95 & 0.87 \\
$\mathbf{0 . 7 5}$ & 41.71 & 0.93 & 38.51 & 0.89 \\
$\mathbf{1}$ & 42.78 & 0.94 & 38.83 & 0.90 \\
$\mathbf{1 . 5}$ & 44.61 & 0.96 & 42.33 & 0.94 \\
$\mathbf{2}$ & 46.51 & 0.97 & 43.29 & 0.95 \\
\hline
\end{tabular}

\begin{tabular}{lllll}
\hline $\begin{array}{l}\text { Rc } \\
\text { (bpp) }\end{array}$ & $\begin{array}{l}\text { CDF9/7 } \\
\text { (filter bank)+SPIHT }\end{array}$ & $\begin{array}{l}\text { CDF9/7(lifting) } \\
\text { +EZW }\end{array}$ \\
\cline { 2 - 5 } & & & & \\
\cline { 2 - 5 } $\mathbf{0 . 1 2 5}$ & 32.90 & 0.74 & 32.40 & 0.68 \\
$\mathbf{0 . 2 5}$ & 33.55 & 0.79 & 33.02 & 0.70 \\
$\mathbf{0 . 5}$ & 35.70 & 0.80 & 35.64 & 0.78 \\
$\mathbf{0 . 7 5}$ & 36.89 & 0.84 & 36.70 & 0.82 \\
$\mathbf{1}$ & 37.65 & 0.88 & 37.32 & 0.87 \\
$\mathbf{1 . 5}$ & 39.88 & 0.89 & 39.25 & 0.88 \\
$\mathbf{2}$ & 41.32 & 0.93 & 40.90 & 0.92 \\
\hline
\end{tabular}

The comparison in terms of image quality for the four algorithms is given by the PSNR and MSSIM curves represented in figures 8 and 9 .

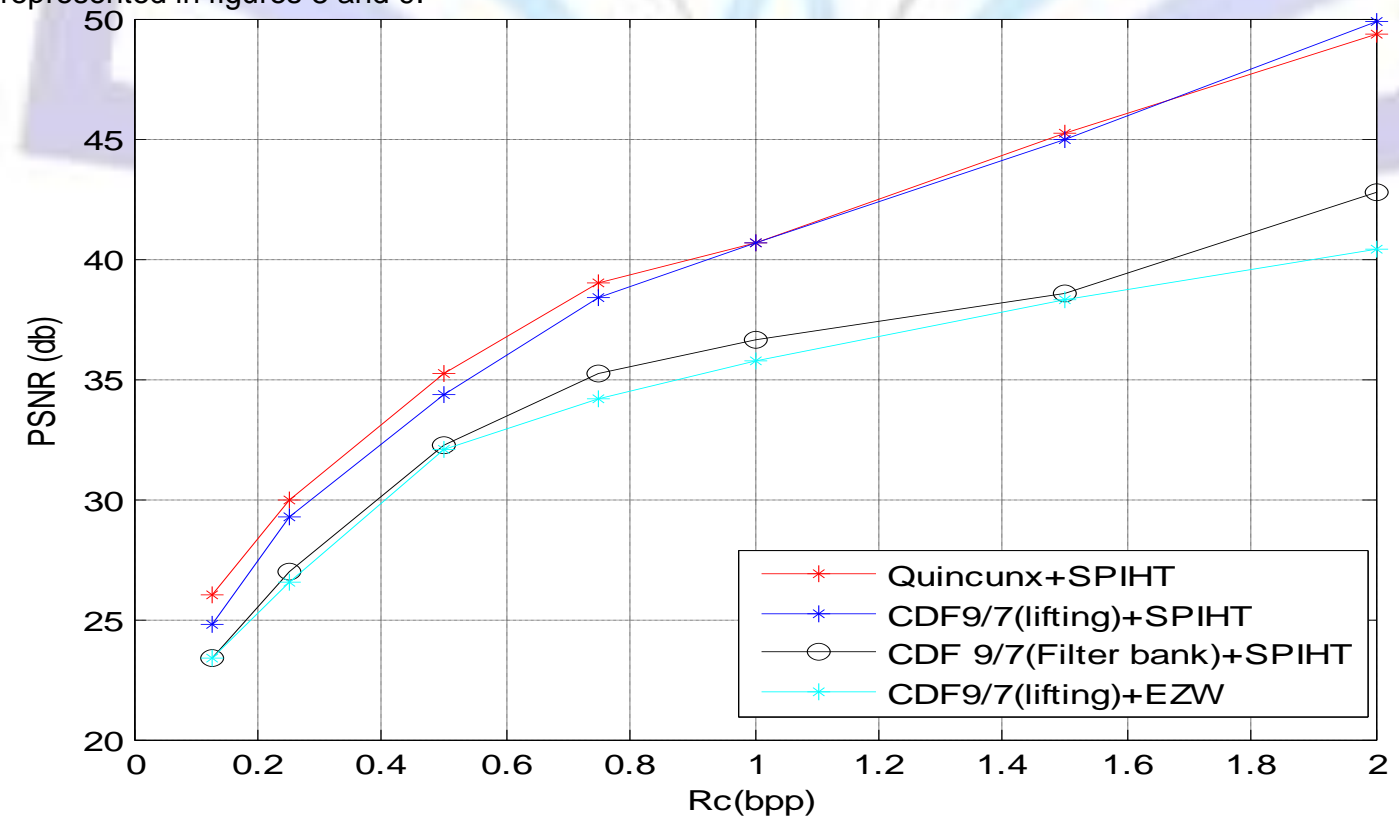

Fig. 8. PSNR variation using different methods 


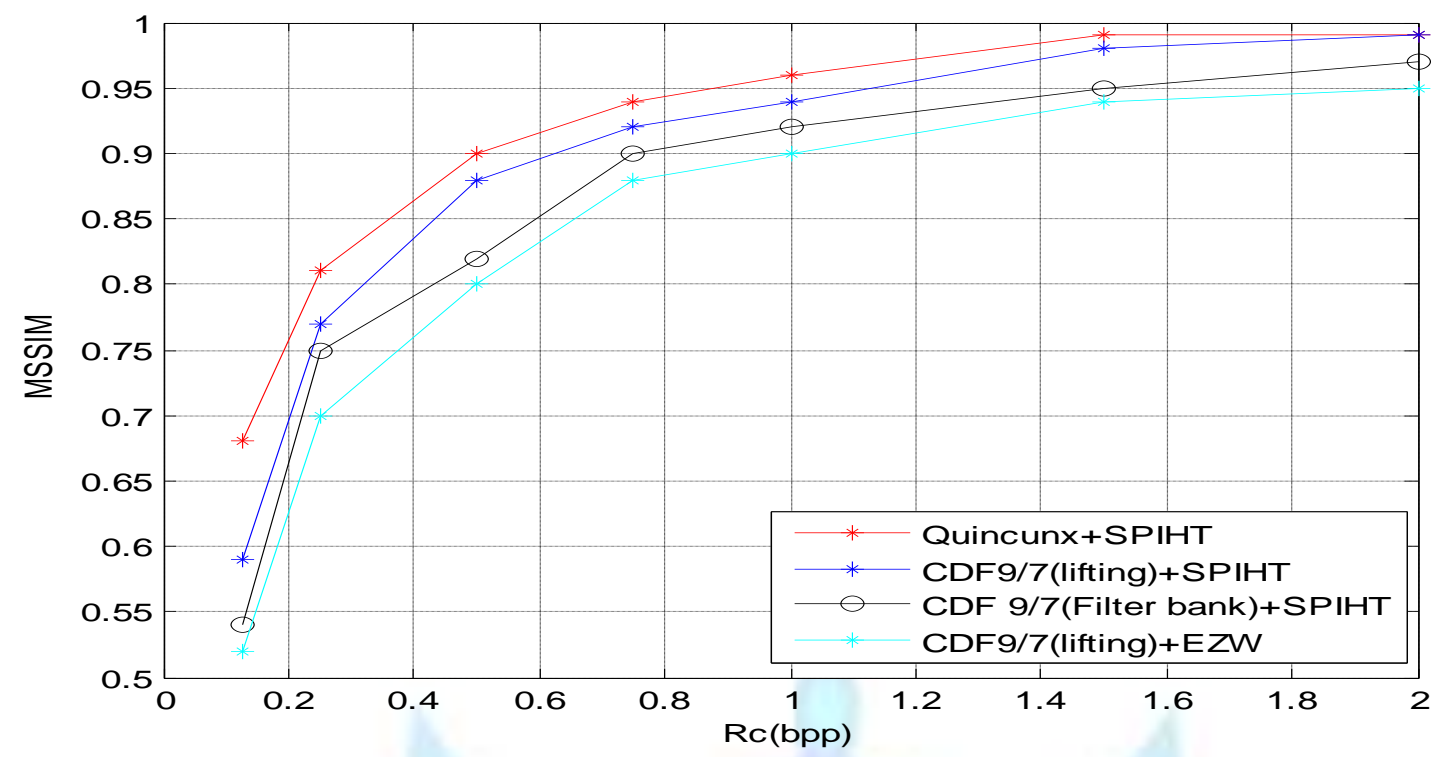

Fig. 9. MSSIM variation using different methods

Comparing the different values of PSNR and MSSIM, we show clearly the efficiency of our algorithm in terms of compressed image quality for the low bit-rate.

This study was subsequently generalized to a set of medical images of the GE Medical Systems database. The following figure (Fig.10) presents the results obtained after application of different algorithms on an axial slice of body imaging. These results are obtained with a 0.5-bpp bit-rate ; this study was subsequently generalized to a set of medical images of the GE Medical Systems database. The Fig. 11 presents the results obtained after application of our algorithm on various slices presented in Fig.10. These results are obtained with a $0.5 \mathrm{bpp}$ bite-rate with Time Computational is obtained $30 \mathrm{~s}$. We note that our algorithm is adapted for the color medical image compression.

We can observe that compression degrades to a lessen extent the image structure for a low compression bit-rate. However, for high compression bitrate, our algorithm better safe guards the various image structures.

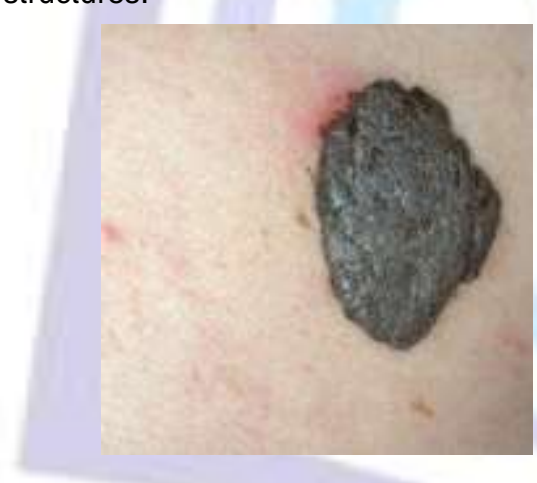

Fig. 10. Original color image: Benign

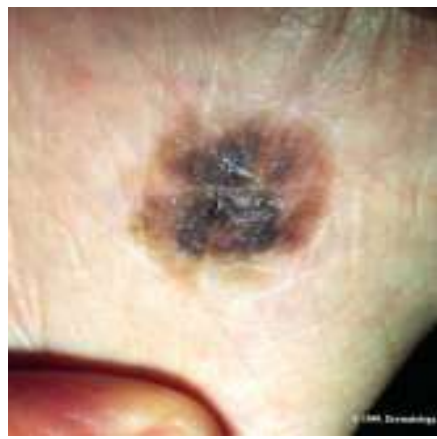

Tc: Time

Fig. 11. Original color image: melanoma

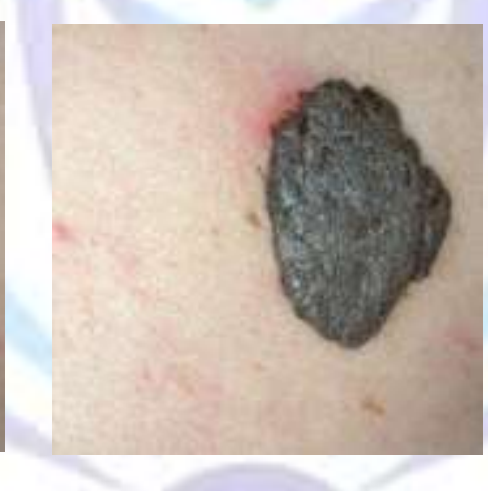

Quincunx wavelet + SPIHT

$P S N R=39.09 \mathrm{~dB}$,

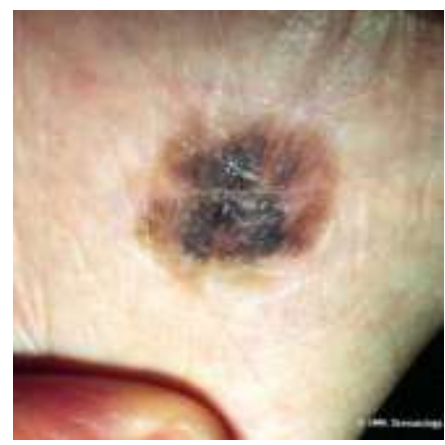

Quincunx wavelet + SPIHT

$P S N R=39.71 \mathrm{~dB}$,

$\mathrm{MSSIM}=0.949, \mathrm{TC}=28.38 \mathrm{~s}$

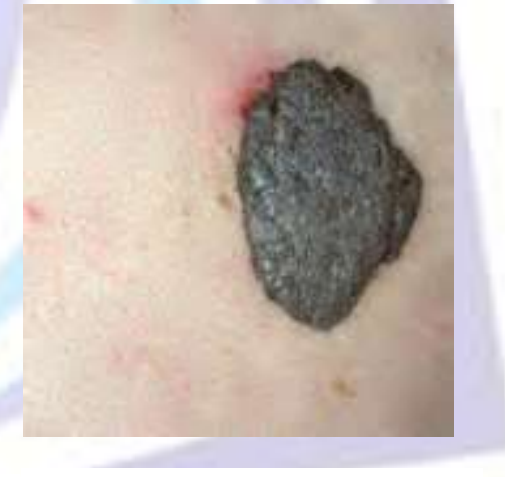

CDF9/7 (lifting sheme) + SPIHT

$P S N R=34.0942 \mathrm{~dB}$,

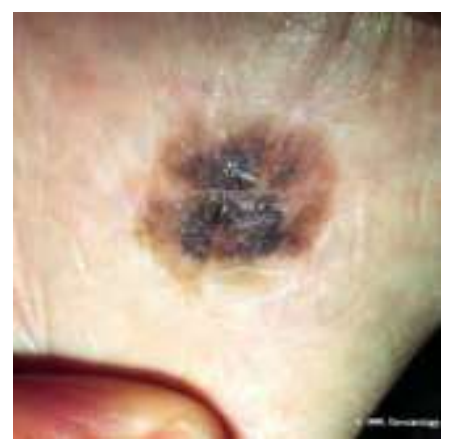

CDF9/7 (lifting sheme) +SPIHT $P S N R=37.00 \mathrm{~dB}$

$\mathrm{MSSIM}=0.933, \mathrm{TC}=64.22 \mathrm{~s}$ 
We can say that our compression algorithm better preserves the different color medical image structures for bitrates higher or equal to $0.5 \mathrm{bpp}$.

\section{Conclusion}

The objective of this paper is undoubtedly the enhancement of medical images quality after the compression step. The latter is regarded as an essential tool to aid diagnosis (storage or transmission) in medical imaging. We used the quincunx wavelet compression coupled with the SPIHT coding. After several applications, we found that this algorithm gives better results than the other compression techniques.

To develop our algorithm, we used various types of color medical images. We have noticed that for 0.5 bpp bit-rate, the algorithm provides very important PSNR and MSSIM values color images. Thus, we conclude that the results obtained are very satisfactory in terms of compression ratio, time computational and compressed image quality.In perspective, we aspire to apply our algorithm to compress video sequences.

\section{References:}

[1]. Chappelier V., "Progressive coding of images by directed wavelet", Phd. Thesis, Rennes1University, December 15th ,2005.

[2]. Mallat S., "Multifrequency channel decompositions of images and wavelet models", IEEE Transaction in Acoustic Speech and Signal Processing, Vol. 37, pp. 2091-2110, Dec. 1989

[3]. Salomon D. "Data ompression", The Complete Reference,Third Edition, Springer.

[4]. Stromme, Oyvind. "On The Applicability of Wavelet Transforms to Image and Video Compression", Ph.D. thesis, University of Strathclyde, February 1999.

[5]. Truchetet F. "Wavelets for digital signal", Hermes Edition, Paris, January 1998.

[6]. Tanaka Y., Ikehara M and Truong Q. N , "A New Combination of 1D and 2D Filter Banks for Effective Multiresolution Image Representation"; IEEE, pp 2820-2823, 2008.

[7]. Vetterli M. and Kovacevé J., "Wavelets and Subband Coding", Upper Saddle River, NJ:Prentice-Hall, 1995.

[8]. Manuela F., Dimitri VD. and Michael U., "An Orthogonal Family of Quincunx Wavelets With Continuously Adjustable Order", IEEE Transactions On Image Processing, Vol. 14, No. 4,APRIL 2005.

[9]. Dimitri VD., Thierry B. and Michael U. "On the Multidimensional Extension of the Quincunx Subsampling Matrix", IEEE Signal Processing Letters, Vol. 12, No. 2, FEBRUARY 2005.

[10].Chen Y. Michael AD. and Wu-Sheng L. "Design of Optimal Quincunx Filter Banks for Image Coding", EURASIP Journal on Advances in Signal Processing, Vol. 2007.

[11].Lee L., Oppenheir V.A., "Proprerties of approximate parks-McClellan filters", IEEE, pp.2165-2168; 1997;

[12].Miaou S.G., Chen S.T. and Chao S.N.,"Wavelet-based lossy-to-lossless medical image compression using dynamic VQ and SPIHTcoding", Biomedical engineering-applications, basis \& communications, Vol. 15 No3, p 235-242, December 2003.

[13].Said A. and Pearlman W. A., "A new fast and efficient image codec based on set partitioning in hierarchical trees", IEEE Trans. Circuits and Systems for Video Technology, Vol. 6, p243 - 250,June 1996.

[14].Yen-Yu C. and Shen-Chuan T., "Embedded medical image compression Using DCT based subband decomposition and modified SPIHT data organization", Proceedings of the Fourth IEEE, (BIBE'04), 2004.

[15].Xiong Z., Ramchandran K., and Orchard M., "Spacefrequency quantization for wavelet image coding", IEEE Trans. Image Processing, Vol. 6, No. 5, pp 677-693, May 1997.

[16].Geisler W. S. and Banks M. S., "Visual performance", in Handbook of Optics (M. Bass, ed.), McGrawHill, 1995.

[17].Watson A. B. and Kreslake L., "Measurement of visual impairment scales for digital video", in Human Vision, Visual Processing, and Digital Display, Proc. SPIE, Vol. 4299, 2001.

[18].Wang Z., Bovik A. C., Sheikh H. R. and Simoncelli E.P, "Image quality assessment: From error visibility to structural similarity", IEEE Transactions on Image Processing, Vol. 13, No. 4, APRIL 2004.

[19].Wang Z. and Bovik A. C, "A universal image quality index", IEEE Signal Processing Letters, Vol. 9, pp.81-84, Mar. 2002.

[20].Buccigrossi R. W. and Simoncelli E. P., "Image compression via joint statistical characterization in the wavelet domain", IEEE Trans. Image processing, Vol. 8, pp. 1688-1701, December 1999.

[21]. Chandler D. M. and Hemami S. S., "Additivity models for suprathreshold distortion in quantized waveletcoded images", in Human Vision and Electronic Imaging VII, Proc. SPIE, Vol. 4662, Jan.2002.

[22].www.GE Medical System.com (database).

[23].www. GE Healthcare.com (database).

\section{AUTHORS' INFORMATION}

1, Genie-Biomedical Laboratory, Electronics Department. Abou bekr Belkaid university,Tlemcen, 13000, Algeria

${ }^{2}$ Bechar University, 08000, Algeria. 


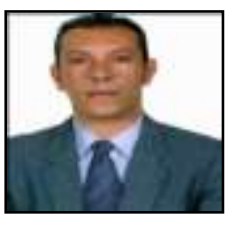

BOUKLI HACENE ISMAIL obtained the Engineer degree in Electronics from university of Tlemcen, Algeria, and then a Magister in Electronic Biomedical from university of Tlemcen, Algeria. His research interests are Image processing, Medical image compression, wavelets transform and optimal encoder.

E-mail: ismaill80@yahoo.fr

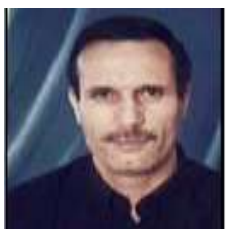

Abdelhafid BESSAID was born in Tlemcen,Algeria. He received the diplom EL-Ing degree from the University of Oran (USTO.Algeria); the Master degree and the PHD from the University of sidi Bel Abbes (Algeria),respectively in 1981,1997 and 2004.He work,since 1996,in the field of Medical Imaging Processing at University of Tlemcen. Algeria

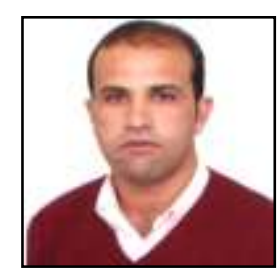

BELADGHAM Mohammed obtained the Engineer degree in Electronics from university of Tlemcen, Algeria, and then a Magister in signals and systems from university of Tlemcen, Algeria. His research interests are Image processing, Medical image compression, wavelets transform and optimal encoder.

e-mail: beladgham@yahoo.fr 